\title{
Habitual coffee drinkers display a distinct pattern of brain functional connectivity
}

\author{
Ricardo Magalhães ${ }^{1,2,3,4} \cdot$ Maria Picó-Pérez $\mathbb{1}^{1,2,3} \cdot$ Madalena Esteves $^{1,2,3} \cdot$ Rita Vieira $\mathbb{1}^{1,2,3} \cdot$ Teresa C. Castanho ${ }^{1,2,3} \cdot$ \\ Liliana Amorim ${ }^{1,2,3} \cdot$ Mafalda Sousa ${ }^{1,2,3} \cdot$ Ana Coelho ${ }^{1,2,3} \cdot$ Henrique M. Fernandes ${ }^{5}$ - Joana Cabral $\mathbb{D}^{1,2,3,5}$. \\ Pedro S. Moreira ${ }^{1,2,3,6} \cdot$ Nuno Sousa $\mathbb{1}^{1,2,3,7}$
}

Received: 19 December 2020 / Accepted: 19 March 2021 / Published online: 20 April 2021

(c) The Author(s) 2021. This article is published with open access

\begin{abstract}
Coffee is the most widely consumed source of caffeine worldwide, partly due to the psychoactive effects of this methylxanthine. Interestingly, the effects of its chronic consumption on the brain's intrinsic functional networks are still largely unknown. This study provides the first extended characterization of the effects of chronic coffee consumption on human brain networks. Subjects were recruited and divided into two groups: habitual coffee drinkers (CD) and non-coffee drinkers (NCD). Resting-state functional magnetic resonance imaging (fMRI) was acquired in these volunteers who were also assessed regarding stress, anxiety, and depression scores. In the neuroimaging evaluation, the CD group showed decreased functional connectivity in the somatosensory and limbic networks during resting state as assessed with independent component analysis. The CD group also showed decreased functional connectivity in a network comprising subcortical and posterior brain regions associated with somatosensory, motor, and emotional processing as assessed with network-based statistics; moreover, $\mathrm{CD}$ displayed longer lifetime of a functional network involving subcortical regions, the visual network and the cerebellum. Importantly, all these differences were dependent on the frequency of caffeine consumption, and were reproduced after NCD drank coffee. CD showed higher stress levels than NCD, and although no other group effects were observed in this psychological assessment, increased frequency of caffeine consumption was also associated with increased anxiety in males. In conclusion, higher consumption of coffee and caffeinated products has an impact in brain functional connectivity at rest with implications in emotionality, alertness, and readiness to action.
\end{abstract}

These authors contributed equally: Ricardo Magalhães, Maria PicóPérez, Madalena Esteves

Supplementary information The online version contains supplementary material available at https://doi.org/10.1038/s41380021-01075-4.

Nuno Sousa

njcsousa@med.uminho.pt

1 Life and Health Sciences Research Institute (ICVS), School of Medicine, University of Minho, Campus de Gualtar, Braga, Portugal

2 ICVS/3B's, PT Government Associate Laboratory, Braga/ Guimarães, Portugal

3 Clinical Academic Center - Braga, Braga, Portugal

\section{Introduction}

Coffee is the most widely consumed beverage, with particular interest for human health in view of its short-term effects on attention, sleep, and memory and its long-term impact on the appearance of different diseases and on healthy span of ageing [1, 2]. Coffee has several constituents able to impact on human health, amongst which stems caffeine, which is the most widely consumed psychostimulant in the world [3]. Despite its widespread use it

4 NeuroSpin, CEA, CNRS, Paris-Saclay University, Gif-surYvette, France

5 Center for Music in the Brain, Department of Clinical Medicine, Aarhus University, Aarhus, Denmark

6 Psychological Neuroscience Lab, CIPsi, School of Psychology, University of Minho, Braga, Portugal

7 P5 Medical Center, Braga, Portugal 
is surprising to note that a thorough characterization of the chronic effects of coffee upon the human brain is still lacking. In the present work we aim to begin addressing that issue.

In the brain, caffeine acts as an antagonist of adenosine $\mathrm{A} 1$ and $\mathrm{A} 2 \mathrm{~A}$ receptors, leading to hyperexcitability of the central nervous system [3,4]. This induces acute effects in diverse domains, such as physical endurance [1, 5], vigilance, dexterity [6], mood [7, 8], memory [9], and cognitive function $[1,8,10]$. There is also evidence that coffee/caffeine intake can normalize anxiety [11], although higher doses of caffeine may be anxiogenic $[1,12]$ by disrupting the HPA axis [13]. On the other hand, epidemiological and animal studies converge in concluding that coffee, caffeine and adenosine receptor antagonists attenuate the burden of neurodegenerative disorders such as Alzheimer's [14], or psychiatric disorders such as depression [15]. Indeed, chronic antagonism of either A1 or A2 receptors seems to induce an upregulation of the former, but not the latter. The resulting altered receptor ratio may explain the shift from the acute psychomotor effects (e.g., attention, vigilance) to the longer-term actions of coffee (e.g., stress resistance, neuroprotection) effects $[4,16]$.

Functional magnetic resonance imaging (fMRI) allows studying, in a noninvasive way, the function of the human brain during execution of different tasks or at rest [17]. So far, most studies using fMRI were focused on measuring the acute effects of caffeine intake in the brain. Briefly, they have reported caffeine-related increases in blood oxygenation-dependent-level (BOLD) signal in different cortical and subcortical areas during a visuomotor task [18]; an impact in working memory and perfusion in elderly subjects $[19,20]$; an increase in BOLD activation in the frontopolar and cingulate cortex during a 2-back verbal working memory task [21, 22]; and a global caffeineinduced increase in brain entropy, possibly representing an increased processing capacity [23]. Very few studies, however, were performed to study the acute effects of caffeine in functional connectivity (FC) at rest [24, 25]. Those few studies reveal a general trend for a caffeineinduced reduction in FC, associated with neuro-electric power fluctuations as measured through magnetoencephalography and exacerbated anticorrelations. Despite this existing literature, many aspects of the characterization of the impact of caffeine on the brain remain unexplored. Critical amongst these is the characterization of the chronic effects of habitual coffee and caffeine consumption upon the functional architecture of the brain. We are only aware of a single study that touched on this subject [26]. That work revealed an association between different habits of coffee consumption and the magnitude of BOLD signals in the visual cortex; however, it did not address possible effects on the functional connectome or resting state networks. Pursuit of the latter can present significant challenges in finding and recruiting participants with sufficient variation in consumption habits and who are willing to undergo necessary, even if short, abstinence procedures.

To tackle this gap, herein we will use whole brain approaches [27-29], as well as the study of brain functional dynamics [30] to compare FC and its dynamics between habitual and non-habitual coffee consumers. In addition, and because of the potential anxiogenic and HPA-disrupting role of caffeine, measures of psychological state (depression, anxiety, and stress) will also be acquired, in order to explore the potential association of habitual coffee consumption with these variables.

\section{Methods}

\section{Subject recruitment and assessment}

Participants were recruited through advertisement on the Institute's social media, institutional e-mail, and press releases distributed among Portuguese local and national newspapers. Exclusion criteria included the presence of neurological or psychiatric disorders, habitual consumption of mind-altering substances, and the inability to undergo MRI. Two experimental groups were created according to participants' coffee consumption habits: coffee drinkers (CD), who drank a minimum of one cup of caffeinated coffee per day; and noncoffee drinkers (NCD), who had no habits of regular consumption of coffee (less than one cup per week). Consumption of coffee as well as other caffeinated products was confirmed in a structured interview. Participants were instructed to abstain from caffeinated products for $3 \mathrm{~h}$ before the assessment, in order to avoid acute influence of caffeine. Fifty-six subjects were recruited ( $32 \mathrm{CD}$ and $24 \mathrm{NCD}$ ). One participant from the $\mathrm{CD}$ group was excluded due to imaging artifacts, rendering a final sample of $31 \mathrm{CD}$ and $24 \mathrm{NCD}$. Characterization of subjects was done in two (CD) or three (NCD) parts within a $3 \mathrm{~h}$ time-period: participants were first interviewed by a certified psychologist. This was followed by an MRI scanning session, and, in the case of the NCD, the first scanning session was followed by ingestion of coffee (Nespresso ${ }^{\circ}$ Ristretto, $\sim 50 \mathrm{cc}$ ) before a rs-fMRI scan $\sim 30 \mathrm{~min}$ thereafter. During the interview, the following data were gathered: demographic data; habits of coffee and other caffeinated products consumption; and assessment of depression, anxiety, and stress scores through the Depression, Anxiety and Stress Scales (DASS-21, [31, 32]).

\section{Demographic and psychological data analysis}

CD and NCD groups were compared in terms of sociodemographic variables, frequency of consumption of 
caffeinated products, and psychological variables. Since the variables did not follow a normal distribution, nonparametric tests were applied (Wilcoxon test). Moreover, multiple regression analyses were performed, aiming to determine the association between daily consumption of caffeinated products such as coffee, tea, chocolate, etc. $(0=<1 /$ day; $1=1 /$ day; $2=2 /$ day; $3=3$ or more/day) and the psychological data measured with the DASS-21 questionnaire (controlled for sex, age, and education), independently of the groups. These analyses were performed on Matlab2020a software (The Mathworks, Inc.) and $p<0.05$ was considered the threshold for statistical significance. Linear regression representations were generated in Prism 7 software (GraphPad Software, Inc.).

\section{MRI brain imaging}

Magnetic resonance imaging scans were conducted using a Siemens Verio 3T (Siemens, Erlangen, Germany) located in Hospital de Braga (Braga, Portugal) using a 32-channel head antenna. The scanning session included as an anatomical acquisition a T1-weighted sagittal magnetization-prepared rapid acquisition with gradient echo $(\mathrm{TE} / \mathrm{TR}=2420 / 4.12 \mathrm{~ms}$, $\mathrm{FA}=9^{\circ}, 1 \mathrm{~mm}^{3}$ isometric voxel size, Field-of-View $=176 \times$ $256 \times 256 \mathrm{~mm}^{3}$ ). The resting-state fMRI (rs-fMRI) acquisition used a multi-band echo planar imaging sequence, CMRR EPI 2D (R2016A, Center for Magnetic Resonance Research, University of Minnesota, Minnesota, USA [33-35]) sensitive to fluctuations in the BOLD contrast $(\mathrm{TR} / \mathrm{TE}=1000 / 27 \mathrm{~ms}$, $\mathrm{FA}=62^{\circ}, 2 \mathrm{~mm}^{3}$ isometric voxel size, 64 axial slices over an in plane matrix of $100 \times 100$ ). The rs-fMRI acquisition had a duration of $7.5 \mathrm{~min}$, during which subjects were instructed to remain with their eyes closed, relaxed, and let their minds wander freely.

\section{Preprocessing of MRI data}

MRI results included in this manuscript were preprocessed using fMRIPrep 1.4.1 ([36]; RRID:SCR_016216), which is based on Nipype 1.2.0 ([37, 38]; RRID:SCR_002502). A full description of the preprocessing pipeline can be found in the Supplementary material.

\section{Resting-state analysis}

\section{Independent component analysis}

Resting-state network (RSN) maps were analyzed voxelwise through a probabilistic independent component analysis (ICA) as implemented in Multivariate Exploratory Linear Optimized Decomposition into Independent Components, distributed with FSL [39]. For further details check the Supplementary material.
The RSNs FC was compared between CD and NCD groups, using a nonparametric permutation procedure implemented in the randomize tool from FSL [40]. Threshold-free cluster enhancement (TFCE) was used to detect widespread significant differences and control the family-wise error rate (FWE-R) at $\alpha=0.05$. In total, 5000 permutations were performed.

\section{Static functional connectomics analysis}

To assess differences between the two groups in the functional connectome, the mean time series of the 268 regions of the Shen Atlas [41] were extracted. The Pearson correlation between time series, followed by Fisher r-to-Z transformation, were calculated to obtain matrices of FC for each subject. To overcome the issue of multiple comparisons induced by the large number of connections in the network, we applied the network-based statistics (NBS) approach [42]. A total of 5000 permutations were used, together with a FWE corrected network significance of 0.05. For more details check the Supplementary material.

\section{Dynamic functional analysis}

We applied the leading eigenvector dynamics analysis (LEiDA, [30]) approach to study the changes in the functional dynamics associated with habitual caffeine consumption. Instantaneous FC was calculated for each subject at each time point for all 268 regions of interest of the Shen atlas, using the time series extracted for the static analysis. To help visually identify phase locked (PL) states, the overlap between each anatomical region of each state to the 7 Yeo RSN's [43], plus two other labels for the cerebellum and subcortical units, was calculated and anatomical units color coded in accordance to the best match. For more details check the reference paper or the Supplementary material.

\section{Effects of acute coffee consumption and frequency of caffeine consumption}

The significant findings obtained with ICA, NBS, and LEiDA were further explored, aiming to assess the effects of acute coffee consumption in NCD and of frequency of consumption of caffeinated products in both groups. The first were assessed by comparing NCD after coffee consumption with data from CD (independent sample $t$-test) and NCD (before coffee consumption; paired sample $t$-test). The second were evaluated by performing multiple regression analyses following the same approach described for the DASS-21 questionnaire. 


\section{Results}

\section{Demographic analysis}

$\mathrm{CD}$ and NCD groups did not differ in terms of age (range $19-57 ; p=0.28 ; Z=1.09 ; r=0.15$ ) or number of formal years of education (range $12-25 ; p=0.07 ; Z=1.84 ; r=$ $0.25)$. Frequency of consumption of caffeinated products was, as expected, higher in the CD group $(p<0.001 ; Z=$ $6.17 ; r=0.83$ ). Sex distribution was not significantly different between groups $\left(\chi^{2}(1, N=55)=0.52, p=0.42\right)$, despite the $\mathrm{CD}$ group presenting a slightly higher proportion of males $(41.94 \%)$ in comparison with the NCD group (33.33\%). Descriptive statistics can be found in Table 1 .

\section{Effect of habitual caffeine consumption on rs-fMRI data}

\section{Independent components analysis}

Thirty components were obtained from the probabilistic ICA of CD and NCD (before consuming coffee). Fifteen of these components were found to be representative of the most typical RSNs. A tendency toward lower FC patterns in the CD group can be seen in most of these networks (see Supplementary Fig. 1). Despite this, we only found significant FWE-R TFCE corrected between-group differences in two of them, namely, in the somatosensory network and the limbic network (Fig. 1). Regarding the somatosensory network, NCD presented a pattern of higher connectivity with the right precuneus (MNI coordinates $=30,-72,38 ; 7$ voxels; peak $t$ value $=4.4$ ). Moreover, for the limbic network, NCD had higher FC in the right insula compared to $\mathrm{CD}$ (MNI coordinates $=42,-12,2 ; 4$ voxels; peak $t$ value $=5.09)$. Of note, these effects were also linearly associated with the caffeinated products' frequency of consumption.

Table 1 Descriptive statistics of demographic data.

\begin{tabular}{|c|c|c|c|c|c|c|c|}
\hline & \multicolumn{2}{|c|}{$\begin{array}{l}\text { Coffee } \\
\text { drinkers } \\
(n=31 ; \\
42 \% \text { male })\end{array}$} & \multicolumn{2}{|c|}{$\begin{array}{l}\text { Non- } \\
\text { coffee } \\
\text { drinkers } \\
(n=24 ; \\
33 \% \text { male) }\end{array}$} & \multicolumn{3}{|c|}{ Statistical analysis } \\
\hline & Med & IQR & Med & IQR & $p$ & $Z$ & $r$ \\
\hline Age & 28.0 & 16.5 & 29.5 & 12.5 & 0.28 & 1.09 & 0.15 \\
\hline Education & 17.0 & 2.0 & 16.0 & 4.0 & 0.07 & 1.84 & 0.25 \\
\hline $\begin{array}{l}\text { Frequency of } \\
\text { consumption }\end{array}$ & 3.0 & 1.0 & 0.0 & 0.0 & $<0.001 *$ & 6.17 & 0.83 \\
\hline
\end{tabular}

Median (Med) and interquartile range (IQR) of age, years of formal education, and frequency of caffeinated drinks' consumption for coffee drinkers and non-coffee drinkers.

*Statistically significant between-group differences at $p<0.05$.
Negative correlations were found for both right precuneus $\left(p=0.003 ; \beta=-1.433\right.$; adjusted $R^{2}=0.162 ;$ Fig. $\left.1 \mathrm{~B}\right)$ and right insula $\left(p<0.001 ; \beta=-2.384\right.$; adjusted $R^{2}=0.267$; Fig. 1B). Detailed statistics can be found in Supplementary Table 1.

Importantly, the group differences described were reduced after NCD drank coffee (see Fig. 1C; somatosensory network: pre vs post NCD $t$ value $=1.86, p=0.075$, post NCD vs CD $t$ value $=-2.89, p=0.006$; limbic network: pre vs post $\mathrm{NCD} t$ value $=3.88, p<0.001$, post $\mathrm{NCD}$ vs $\mathrm{CD} t$ value $=-1.46, p=0.15$ ). This points to a potential causality link between coffee drinking and the abovedescribed changes in lower connectivity in the somatosensory and in the limbic networks.

\section{Connectomics analysis}

From the connectomics analysis done using NBS, a single network of significantly higher connectivity was found in the NCD group (pre-coffee) between the thresholds of 0.005 and 0.0005 (for statistics of all thresholds see Supplementary Table 2). For ease of visualization, we present only the results found at the highest significant threshold of $p=0.0005$ ( $t$ (threshold) $=3.71, \mathrm{df}=54, p$ (network) $=0.043$, Hedge's $g=1.08$ (large effect size), 24 nodes, 46 edges; Fig. 2A). The full list of nodes with significant different edges between groups across all thresholds can be found in Supplementary Table 3. Of these we highlight the Thalamus (nodes \#262 and \#126), the Cerebellum (left anterior Culmen \#245 and bilateral Tonsils \#238 and \#119), the right Postcentral Gyrus (\#33), the left Middle Temporal Gyrus (\#197), the left Precentral Gyrus (\#160), and the bilateral Caudate (\#260 and \#122) and Putamen (\#124 and \#261) as having the most strongly affected connections within the network.

When observing the average network connectivity from this network, NCD post-coffee drink displayed a significant reduction in connectivity (Fig. 2B), leading to a profile more similar to $\mathrm{CD}(p=0.037, t=2.13, \mathrm{df}=54)$ than to NCD pre-coffee drink $\left(p=1.3 \times 10^{-7}, t=7.4, \mathrm{df}=23\right)$. NBS mean FC was negatively associated with frequency of caffeine consumption $(p<0.001 ; \beta=-0.101$; adjusted $R^{2}=0.506$; Fig. 2C). Detailed statistics can be found in Supplementary Table 1 .

\section{Dynamic FC}

From the dynamic FC analysis, one functional subsystem (Fig. 3A, PL state 4) was found to last significantly longer in $\mathrm{CD}$ (Fig. 3B, $17.95 \pm 18.32 \mathrm{~s}$ ) compared to pre-coffee NCD $(8.95 \pm 6.13 \mathrm{~s})$ surviving correction for multiple comparisons with a corrected $p=0.038$ and a medium 
A
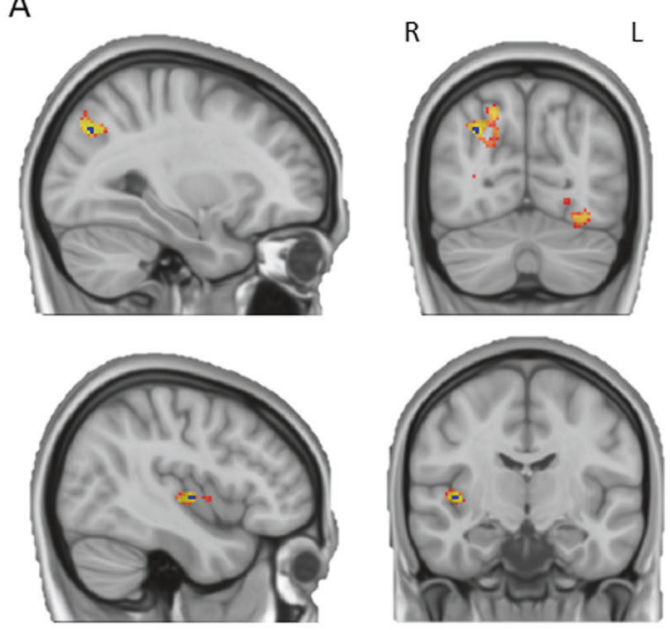

C

ICA right precuneus FC

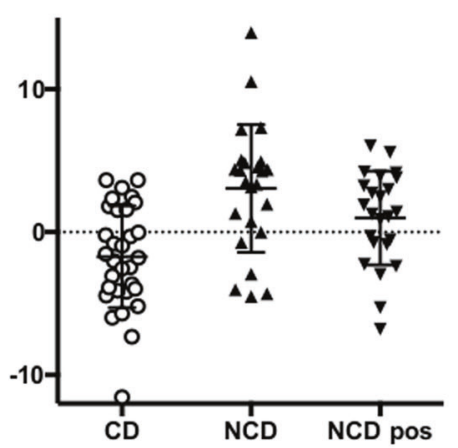

Fig. 1 Results from ICA. A Sagittal, coronal, and axial view of the clusters showing significant between-group differences in the connectivity between the somatosensory network and the right precuneus (top) and the limbic network and the right insula (bottom). The FWE-R TFCE corrected clusters are shown in dark blue overlaid over a more extended non-significant after multiple comparison correction cluster
B

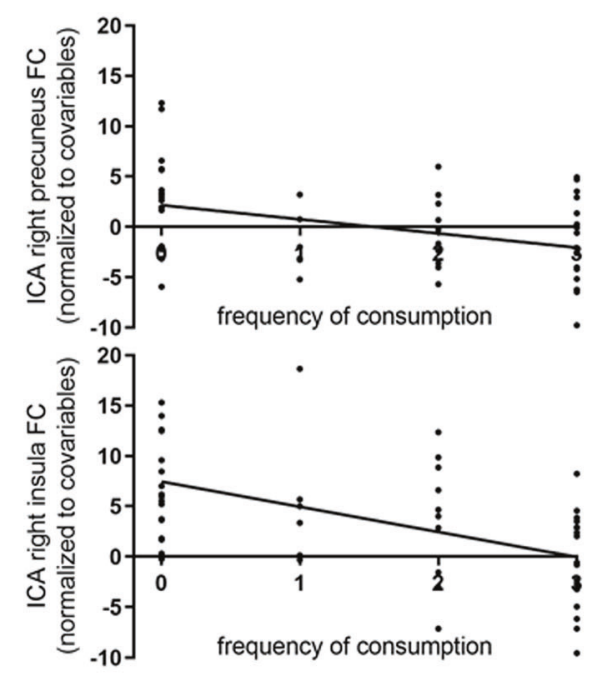

ICA right insula FC

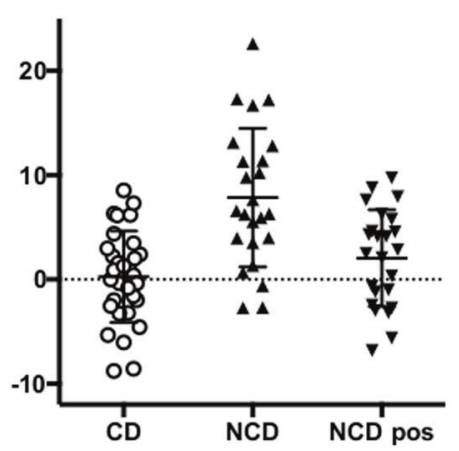

in hot color scale scheme, for visualization purposes. B Associations of frequency of consumption of caffeinated beverages with the mean $\mathrm{FC}$ of the right precuneus and the right insula. $\mathbf{C}$ Scatter plots showing the mean FC of the right precuneus and the right insula for the NCD before drinking coffee (NCD), the NCD after drinking coffee (NCD pos), and the CD.

Fig. 2 Network with reduced functional connectivity (FC) in habitual coffee drinkers as uncovered by NBS. A Sagittal, coronal, and axial view of the network with nodes and edges colored in red-yellow color scheme representing the statistical $t$ value of the difference between groups. B Scatter plot of the mean FC within the significant network for each experimental group. C Associations of frequency of consumption of caffeinated beverages with the mean FC of the network found in NBS.
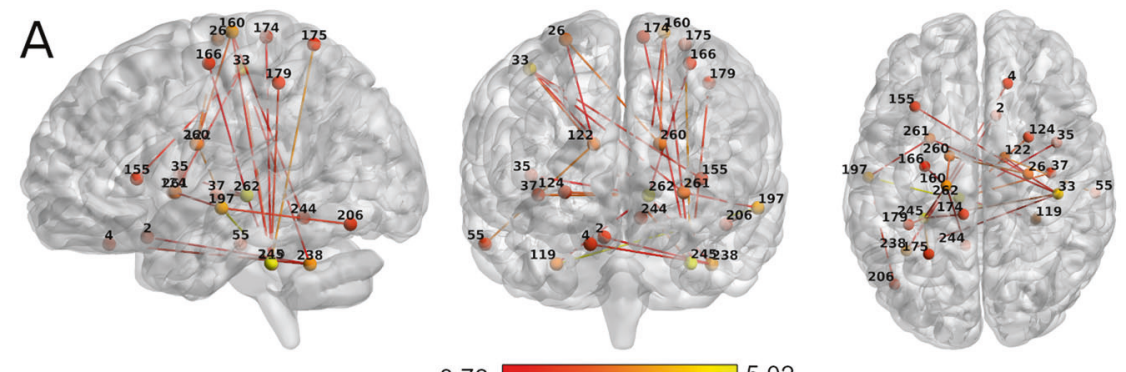

3.72 5.02
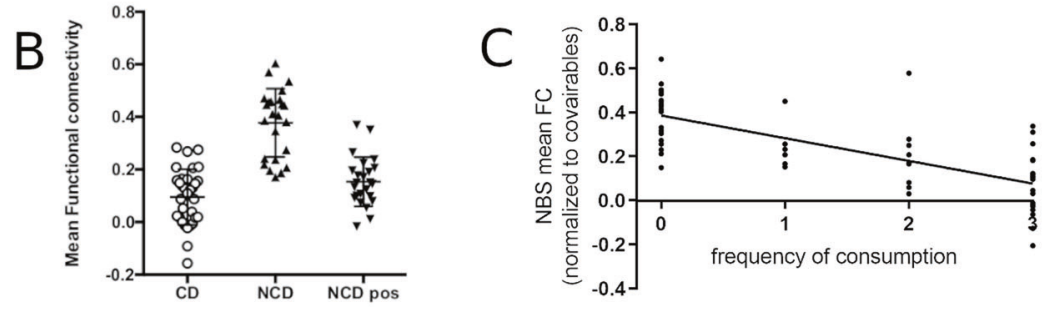
A
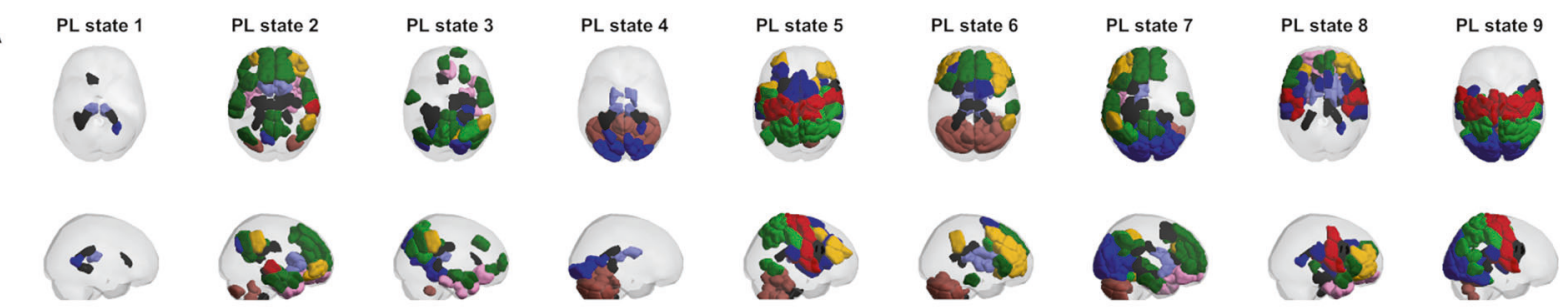

B
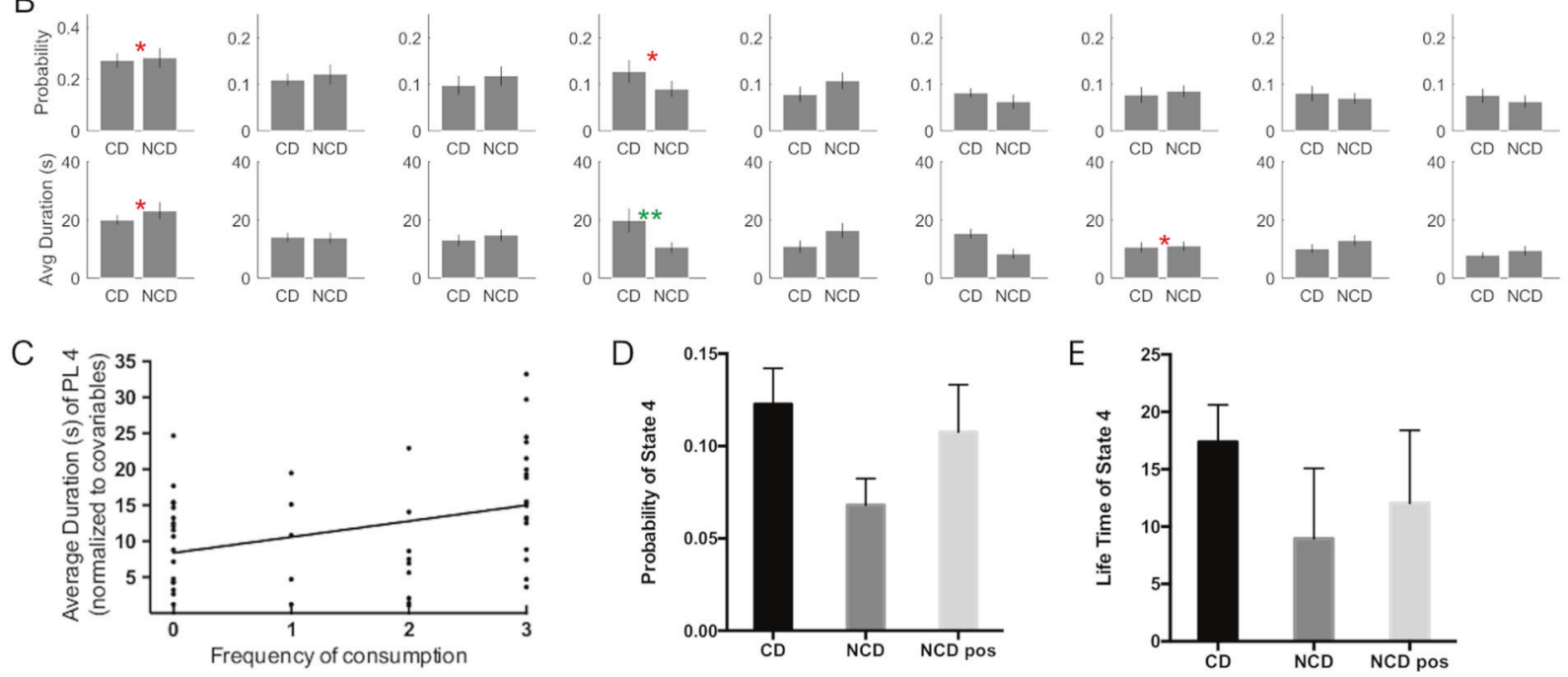

F

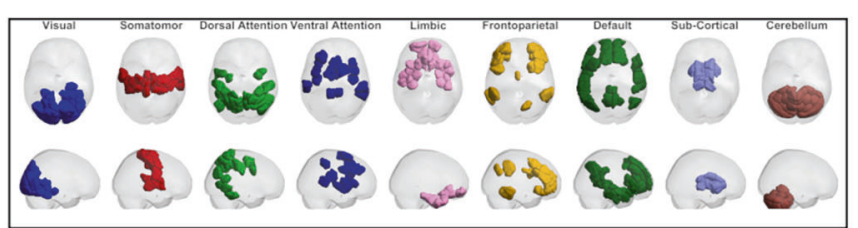

Fig. 3 LEiDA results for $k=9$ of the k-means clustering algorithm and group comparisons of state probability and life time between coffee and non-coffee drinkers. A sagittal and axial views representing the state anatomical areas of each phase locked (PL) state. B Bar plot representing the group differences between coffee and noncoffee drinkers. Differences of $p<0.05$ are indicated in red, while multiple comparison surviving effects are indicated in green.
C Associations of frequency of consumption of caffeinated beverages with the average duration (in seconds) of PL state 4. D Bar plot of the probability of state 4 for the $\mathrm{CD}$, NCD, and NCD post caffeine consumption groups. E Life time of state 4 for the $\mathrm{CD}, \mathrm{NCD}$, and NCD post caffeine consumption groups. F Colored labels used to match each anatomical area of the PL states to different resting state networks. effect size with Hedge's $g=0.62$. No BOLD phase-locking state was found to significantly differ in terms of probability of occurrence (see Supplementary Table 4 for all $p$ values for all partition models).

This BOLD phase-locking state, corresponding to the fourth most probable state when partitioning the data into nine states, comprises a large number of nodes in the cerebellum, visual network as well as several subcortical nodes such as the bilateral thalamus and parahippocampal gyrus (mapped and color coded through the reference shown in Fig. 3F). While this was the only result that survived correction for multiple comparisons, it is relevant to note that the equivalent LEiDA state for $k=10$ is just below the threshold ( $p=0.051$, Supplementary Table 4 and Supplementary Figs. 2 and 3 ).
Furthermore, LEiDA lifetime results were positively correlated with frequency of caffeine consumption ( $p=$ $0.012 ; \beta=2.176$; adjusted $R^{2}=0.083$; Fig. $3 C$ ).

After drinking coffee, both the lifetime and the probability of this state in NCD became closer to the values observed in $\mathrm{CD}$, with the probability not being significantly different from CD $(p=0.5, t=0.67, \mathrm{df}=$ $54)$, while being significantly higher than NCD precoffee $(p=0.037, t=2.31, \mathrm{df}=23$, Fig. 3D). For the life time of state 4 , post-coffee drink NCD were not significantly different from $\mathrm{CD}(p=0.177, t=1.37$, $\mathrm{df}=54)$ nor the pre-drink NCD $(p=0.107, t=1.68$, $\mathrm{df}=23$, Fig. 3E). All results across the different $k$ 's can be found in Supplementary Figs. 2 and 3 and Supplementary Table 4 . 
A

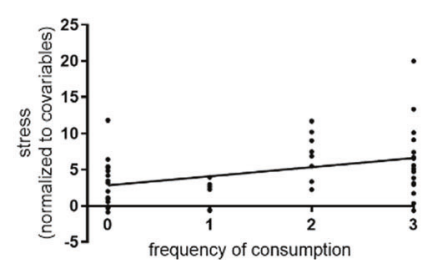

B

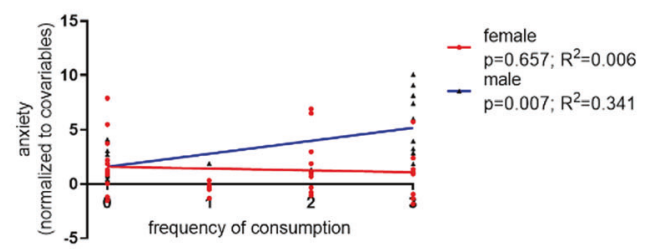

C

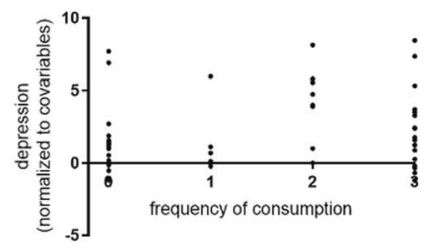

Fig. 4 Graphical depiction of the linear regressions associating frequency of consumption of caffeinated products with self-report variables. Associations of frequency of consumption of caffeinated

\section{Effect of habitual caffeine consumption on psychological data}

The association between coffee consumption and stress, anxiety, and depression (DASS-21) was assessed. When comparing $\mathrm{CD}$ and NCD groups, only stress was significantly different between groups (stress $-p=0.025$; $Z=2.237 ; \quad r=0.307 ;$ anxiety $-p=0.851 ; Z=-0.188$; $r=-0.026$; depression $-p=0.085 ; Z=1.724 ; r=0.237$ ), with CD showing higher levels of stress than NCD (median $(\mathrm{Med})=6.0$; interquartile range $(\mathrm{IQR})=6.0 \mathrm{vs} \mathrm{Med}=4.0$; $\mathrm{IQR}=4.0$, respectively). Of notice, particular items of the DASS-21 Stress subscale that can be related to arousal were increased in CD. Items \#1 and \#12, which measure difficulty to relax, presented statistically significant differences ( $p=0.007$, Mann-Whitney test), while item \#8, that relates to nervous arousal, presented a trend in the same direction $(p=0.083)$. Interestingly, item \#7 (Anxiety subscale), that is associated with skeletal musculature, despite not achieving a statistically significant difference between groups, tended to be lower in $\mathrm{CD}(p=0.113)$, suggesting a segregation between the motor and arousal loops.

When assessing the effects of frequency of caffeine consumption in self-reported variables (controlling for sex, age, and education), the positive correlation with stress was maintained ( $p=0.004 ; \beta=1.292$; adjusted $R^{2}$ $=0.135$; Fig. $4 \mathrm{~A}$ ). Moreover, a sex by anxiety interaction was found $\left(p=0.023 ; \beta=0.683\right.$; adjusted $R^{2}=0.085$; Fig. 4B), which seems to be driven by a positive correlation in males. No significant effects were found for the depression subscale $\left(p=0.128 ; \beta=0.450\right.$; adjusted $R^{2}=$ 0.108 ; Fig. 4C). Detailed statistics can be found in Supplementary Table 1.

\section{Discussion}

Herein we describe for the first time the effects of habitual coffee consumption on the human brain networks. We show that habitual CD have different patterns of FC in comparison with NCD. Our rs-fMRI analysis revealed decreased products with the DASS-21 subscales of stress (A) and anxiety (B), and non-significant association with the depression subscale $(\mathbf{C})$.

FC of the somatosensory and limbic networks in CD that correlated with the frequency of consumption of caffeinated products. Such changes were replicated in NCD after a single coffee, suggesting possible causality between coffee intake and altered patterns of brain network connectivity. Previous studies have described a reduction of similar RSN connectivity after acute caffeine ingestion [25, 44].

Decreased FC in somatosensory and related networks in CD likely represents a more efficient and beneficial pattern of connections with respect to motor control and alertness; importantly this fits our findings of trends of increased scores in CD in the specific items of the DASS-21 scale that measure these dimensions. The other network impacted by coffee intake was the limbic network, which is involved in processing the sensory input from the external and internal environment which, by modulating memory and motivation, determine emotional, autonomic, motor, and cognitive responses [45]. A previous resting-state PET study showed reduced metabolic activity in components of this network after caffeine ingestion [18] and a study using a hedonic fMRI task showed decreased activation in neuronal areas associated with memory and reward [46] in caffeine consumers compared to non-consumers; the present FC data are consistent with those reports.

Analysis of the global functional connectome using NBS revealed a network impacted by the habitual consumption of caffeine. This widespread network of reduced FC comprised cerebellar, subcortical (striatal and thalamic), and motor cortex regions, partially matching previously reported effects of acute caffeine ingestion [24, 25]. Interestingly, there is a clear bilateral involvement of striatal nodes and of the thalamus which, respectively, have the highest densities of A2A and A1 receptors in the brain [47, 48]. The action of caffeine in these regions has an influence on cortico-striatalthalamic and cerebellar-thalamocortical loops that are relevant for a variety of neuronal processes. Thus, the observed decrease in FC at rest in this network in regular caffeineingesting individuals reveals greater segregation of these areas with less inter-regional dependencies, favoring greater efficiency within these loops. It is relevant to note here that, even though $\mathrm{A} 1$ and $\mathrm{A} 2 \mathrm{~A}$ receptors are thought to mediate 
differential actions [49], similar effects were observed in both loops. This likely reflects the fact that fMRI provides proxy aggregate measurements of functional connections among a network of brain areas.

A previous study reported that caffeine increases brain entropy, indicating higher information processing capacity across the cerebral cortex [23]. Our LEiDA analysis revealed a dynamic state involving several cerebellar and subcortical areas, with a longer average lifetime in habitual $\mathrm{CD}$. This network comprises several nodes, including the cerebellum, thalamus, and parahipocampal, lingual, and inferior occipital gyri which are relevant in the context of caffeine consumption - caffeine is known to decrease mind wandering [50] and to increase attention, alertness, and arousal [51]. In fact, the nodes implicated in this network are linked by visual processing; among these, the thalamus is critical for distributing cognitive control [52]. The lingual and inferior occipital gyrus are also implicated in visual processing, while the parahippocampus is involved in memory encoding and retrieval [20,21]; the latter may explain why caffeine reportedly facilitates memory processes [9]. Lastly, evidence of strong $\mathrm{rsFC}$ between the cerebellum, known to be also implicated in sensory processing [53] and a number of sensorial cortices [54], explains the observed increased visual alertness/attention and readiness to sensorial perception among $\mathrm{CD}$ individuals. While similar findings have been previously reported [6], only one other study assessed habitual CD using MRI, and did not characterize changes in FC [26]. Importantly, similarly to the other neuroimaging findings, a common pattern of connectivity dynamics was found in $\mathrm{CD}$ individuals and NCD subjects who drank a single coffee before scanning.

In order to provide a link with other neuropsychologic dimensions, we also assessed our subjects in the DASS- 21 . Interestingly, we observed habitual CD to display increased levels of stress; there was a clear positive association between the indices of stress and the amount of consumption of caffeinated drinks. Interestingly, items of the DASS21 sub-score that showed greater variance between $C D$ vs NCD were those related with difficulty to relax (items \#1 and \#12), and those related to nervous arousal (item \#8), consistent with the common attribution of alertness and arousal to coffee intake. It also deserves to be mentioned that, despite the display of a higher anxiety among CD (particularly in males), there was a decrease in DASS-21 item (\#7) which matches the effects on the skeletal muscles in $\mathrm{CD}$; this, in turn, fits the findings of better segregation of the above-described loops. The present results extend previous studies that described an association between coffee/ caffeine consumption and stress and anxiety [1, 13, 16, 55] and sex $[13,16]$. It is important to note, however, that causality cannot be inferred from our study design. Our results are open to two interpretations: higher coffee/caffeine consumption leads to increased stress and anxiety; or, alternatively, higher stress and anxiety induce higher coffee/ caffeine consumption. Moreover, given that resting-state studies using stress and anxiety samples have shown both decreases and increases in FC [56-58], the possibility that coffee/caffeine consumption elicits decreases in FC or compensates for FC beyond a certain threshold, must also be considered. While the first possibility is in line with studies showing increased anxiety upon both acute caffeine administration in humans $[1,12]$ and prolonged ingestion in rodents [59] reports that greater caffeine consumption under periods of stress may help maintain synaptic homeostasis [60] as well as prevent mood disorders warrant further study in future.

The methodologies applied in the present study do not allow us to draw precise relationships between the psychological and neuroimaging results and the dosage and metabolism of caffeine among individual subjects. To study the individual responses to the acute and chronic effects of caffeinated product intake would be a complex undertaking, requiring subjects to adapt their daily habits and strict abstinence regimens. Based on our experience, recruitment of subjects for a properly balanced study is also difficult since NCD subjects are insufficiently motivated to engage in studies on the actions of caffeine. Nevertheless, we are currently developing alternative strategies that would allow us to deliver calibrated doses of caffeine during fMRI scanning sessions to better discriminate its effects from other factors (e.g., stress). Our future work will also examine inter-individual differences in response to caffeine consumption, the subjective experience of coffee consumption, as well as the influence of additional factors as the consumption of alcohol and tobacco. Despite such gaps, the data presented here represent a contribution to the knowledge of the "caffeinated brain" and how these changes underlie the behavioral effects triggered by coffee intake, with implications for physiological and pathological conditions.

\section{Code availability}

In-house scripts used in the NBS analysis are fully available online at open science framework website (https://osf.io/ qepc8/) and LEiDA scripts at github (https://github.com/jua nitacabral/LEiDA).

Funding This study was funded by the Institute for the Scientific Information on Coffee (ISIC) (ISIC_2017_NS); ISIC did not influence the experimental design or data analysis/interpretation. The laboratory was also supported by the project NORTE-01-0145-FEDER000013 through the Northern Portugal Regional Operational Programme (NORTE 2020), under the Portugal 2020 Partnership Agreement, through the European Regional Development Fund (FEDER). RM, 
MP-P, and ME were supported by post-doctoral grants from the project ISIC_2017_NS. PSM was supported by a fellowship grant from the Fundação para a Ciência e a Tecnologia (FCT; grant number PDE/ $\mathrm{BDE} / 113601 / 2015)$ from the PhD-iHES program. RV was supported by a research fellowship of the project funded by FCT (UMINHO/BI/ 340/2018). AC was supported by a scholarship from the project NORTE-08-5639-FSE-000041 (NORTE 2020; UMINHO/BD/51/ 2017).

\section{Compliance with ethical standards}

Conflict of interest The authors declare no competing interests.

Ethical approval The present study was conducted in accordance with the principles expressed in the Declaration of Helsinki (59th amendment) and was approved by the ethics committee of Hospital de Braga. All participants gave informed written consent after the study aims were explained and were informed of the option to withdraw from the study at any time.

Publisher's note Springer Nature remains neutral with regard to jurisdictional claims in published maps and institutional affiliations.

Open Access This article is licensed under a Creative Commons Attribution 4.0 International License, which permits use, sharing, adaptation, distribution and reproduction in any medium or format, as long as you give appropriate credit to the original author(s) and the source, provide a link to the Creative Commons license, and indicate if changes were made. The images or other third party material in this article are included in the article's Creative Commons license, unless indicated otherwise in a credit line to the material. If material is not included in the article's Creative Commons license and your intended use is not permitted by statutory regulation or exceeds the permitted use, you will need to obtain permission directly from the copyright holder. To view a copy of this license, visit http://creativecommons. org/licenses/by/4.0/.

\section{References}

1. McLellan TM, Caldwell JA, Lieberman HR. A review of caffeine's effects on cognitive, physical and occupational performance. Neurosci Biobehav Rev. 2016;71:294-312.

2. O'Keefe JH, DiNicolantonio JJ, Lavie CJ. Coffee for cardioprotection and longevity. Prog Cardiovascular Dis. 2018;61:38-42.

3. Fredholm BB, Bättig K, Holmén J, Nehlig A, Zvartau EE. Actions of caffeine in the brain with special reference to factors that contribute to its widespread use. Pharmacol Rev. 1999;51:83-133.

4. Ribeiro JA, Sebastião AM. Caffeine and Adenosine. J Alzheimer's Dis. 2010;20:S3-15.

5. Southward K, Rutherfurd-Markwick KJ, Ali A. The effect of acute caffeine ingestion on endurance performance: a systematic review and meta-analysis. Sports Med. 2018;48:1913-28.

6. Killgore WDS, Kamimori GH. Multiple caffeine doses maintain vigilance, attention, complex motor sequence expression, and manual dexterity during $77 \mathrm{~h}$ of total sleep deprivation. Neurobiol Sleep Circadian Rhythms. 2020;9:100051.

7. Sane RM, Jadhav PR, Subhedar SN. The acute effects of decaffeinated versus caffeinated coffee on reaction time, mood and skeletal muscle strength. J Basic Clin Physiol Pharmacol. 2019;30.

8. Smit HJ, Rogers PJ. Effects of low doses of caffeine on cognitive performance, mood and thirst in low and higher caffeine consumers. Psychopharmacology (Berl). 2000;152:167-73.
9. Borota D, Murray E, Keceli G, Chang A, Watabe JM, Ly M, et al. Post-study caffeine administration enhances memory consolidation in humans. Nat Neurosci. 2014;17:201-3.

10. Franceschini S, Lulli M, Bertoni S, Gori S, Angrilli A, Mancarella $\mathrm{M}$, et al. Caffeine improves text reading and global perception. J Psychopharmacol. 2020;34:315-25.

11. Harris A, Ursin H, Murison R, Eriksen HR. Coffee, stress and cortisol in nursing staff. Psychoneuroendocrinology 2007;32:322-30.

12. Bruce M. Anxiogenic effects of caffeine in patients with anxiety disorders. Arch Gen Psychiatry. 1992;49:867.

13. O’Neill CE, Newsom RJ, Stafford J, Scott T, Archuleta S, Levis $\mathrm{SC}$, et al. Adolescent caffeine consumption increases adulthood anxiety-related behavior and modifies neuroendocrine signaling. Psychoneuroendocrinology. 2016;67:40-50.

14. Eskelinen $\mathrm{MH}$, Kivipelto $M$. Caffeine as a protective factor in dementia and Alzheimer's disease. J Alzheimer's Dis. 2010;20: S167-74.

15. Wang L, Shen X, Wu Y, Zhang D. Coffee and caffeine consumption and depression: a meta-analysis of observational studies. Aust NZ J Psychiatry. 2016;50:228-42.

16. Kaster MP, Machado NJ, Silva HB, Nunes A, Ardais AP, Santana $\mathrm{M}$, et al. Caffeine acts through neuronal adenosine A2A receptors to prevent mood and memory dysfunction triggered by chronic stress. Proc Natl Acad Sci USA. 2015;112:7833-8.

17. Soares JM, Magalhães R, Moreira PS, Sousa A, Ganz E, Sampaio A, et al. A Hitchhiker's guide to functional magnetic resonance imaging. Front Neurosci. 2016;10:515.

18. Park CA, Kang CK, Son YD, Choi EJ, Kim SH, Oh ST, et al. The effects of caffeine ingestion on cortical areas: functional imaging study. Magn Reson Imaging. 2014;32:366-71.

19. Haller S, Rodriguez C, Moser D, Toma S, Hofmeister J, Sinanaj I, et al. Acute caffeine administration impact on working memoryrelated brain activation and functional connectivity in the elderly: a BOLD and perfusion MRI study. Neuroscience. 2013;250:364-71.

20. Klaassen EB, de Groot RHM, Evers EAT, Snel J, Veerman ECI, Ligtenberg AJM, et al. The effect of caffeine on working memory load-related brain activation in middle-aged males. Neuropharmacology. 2013;64:160-7.

21. Haller S, Montandon ML, Rodriguez C, Moser D, Toma S, Hofmeister $\mathrm{J}$, et al. Caffeine impact on working memory-related network activation patterns in early stages of cognitive decline. Neuroradiology. 2017;59:387-95.

22. Koppelstaetter F, Poeppel TD, Siedentopf CM, Ischebeck A, Verius M, Haala I, et al. Does caffeine modulate verbal working memory processes? An fMRI study. NeuroImage. 2008;39:492-9.

23. Chang D, Song D, Zhang J, Shang Y, Ge Q, Wang Z. Caffeine caused a widespread increase of resting brain entropy. Sci Rep. 2018;8:2700

24. Tal O, Diwakar M, Wong CW, Olafsson V, Lee R, Huang MX, et al. Caffeine-induced global reductions in resting-state BOLD connectivity reflect widespread decreases in MEG connectivity. Front Hum Neurosci. 2013;7:63.

25. Wong CW, Olafsson V, Tal O, Liu TT. Anti-correlated networks, global signal regression, and the effects of caffeine in resting-state functional MRI. NeuroImage. 2012;63:356-64.

26. Laurienti PJ, Field AS, Burdette JH, Maldjian JA, Yen Y-F, Moody DM. Dietary caffeine consumption modulates fMRI measures. NeuroImage. 2002;17:751-7.

27. Bullmore E, Sporns O. Complex brain networks: graph theoretical analysis of structural and functional systems. Nat Rev Neurosci. 2009;10:186-98.

28. Deco G, Kringelbach ML. Great expectations: using whole-brain computational connectomics for understanding neuropsychiatric disorders. Neuron. 2014;84:892-905. 
29. Sporns O, Chialvo D, Kaiser M, Hilgetag C. Organization, development and function of complex brain networks. Trends Cogn Sci. 2004;8:418-25.

30. Cabral J, Vidaurre D, Marques P, Magalhães R, Silva Moreira P, Miguel, et al. Cognitive performance in healthy older adults relates to spontaneous switching between states of functional connectivity during rest. Sci Rep. 2017;7:5135.

31. Lovibond PF, Lovibond SH. The structure of negative emotional states: comparison of the Depression Anxiety Stress Scales (DASS) with the beck depression and anxiety inventories. Behav Res Ther. 1995;33:335-43.

32. Pais-Ribeiro JL, Honrado A, Leal I. Contribuição para o Estudo da Adaptação Portuguesa das Escalas de Ansiedade, Depressão e Stress (EADS) de 21 itens de Lovibond e Lovibond. Psic Saúde Doenç. 2004;5:229-39.

33. Feinberg DA, Moeller S, Smith SM, Auerbach E, Ramanna S, Glasser MF, et al. Multiplexed echo planar imaging for subsecond whole brain FMRI and fast diffusion imaging. PLoS ONE. 2010;5:e15710.

34. Moeller S, Yacoub E, Olman CA, Auerbach E, Strupp J, Harel N, et al. Multiband multislice GE-EPI at 7 tesla, with 16 -fold acceleration using partial parallel imaging with application to high spatial and temporal whole-brain fMRI. Magn Reson Med. 2010;63:1144-53.

35. Xu J, Moeller S, Auerbach EJ, Strupp J, Smith SM, Feinberg DA, et al. Evaluation of slice accelerations using multiband echo planar imaging at 3T. NeuroImage. 2013;83:991-1001.

36. Esteban O, Markiewicz CJ, Blair RW, Moodie CA, Isik AI, Erramuzpe A, et al. fMRIPrep: a robust preprocessing pipeline for functional MRI. Nat Methods. 2019;16:111-6.

37. Gorgolewski KJ, Esteban O, Ellis DG, Notter MP, Ziegler E, Johnson H, et al. Nipype: a flexible, lightweight and extensible neuroimaging data processing framework in Python. 0.13.1. Zenodo. 2017.

38. Gorgolewski K, Burns CD, Madison C, Clark D, Halchenko YO, Waskom ML, et al. Nipype: a flexible, lightweight and extensible neuroimaging data processing framework in python. Front Neuroinform. 2011;5:13.

39. Beckmann CF, Smith SM. Probabilistic independent component analysis for functional magnetic resonance imaging. IEEE Trans Med Imaging. 2004;23:137-52.

40. Winkler AM, Ridgway GR, Webster MA, Smith SM, Nichols TE. Permutation inference for the general linear model. NeuroImage. 2014;92:381-97.

41. Shen X, Tokoglu F, Papademetris X, Constable RT. Groupwise whole-brain parcellation from resting-state fMRI data for network node identification. NeuroImage. 2013;82:403-15.

42. Zalesky A, Fornito A, Bullmore ET. Network-based statistic: identifying differences in brain networks. NeuroImage. 2010;53:1197-207.

43. Thomas Yeo BT, Krienen FM, Sepulcre J, Sabuncu MR, Lashkari D, Hollinshead M, et al. The organization of the human cerebral cortex estimated by intrinsic functional connectivity. J Neurophysiol. 2011;106:1125-65.
44. Rack-Gomer AL, Liu TT. Caffeine increases the temporal variability of resting-state BOLD connectivity in the motor cortex. NeuroImage. 2012;59:2994-3002.

45. McLachlan RS. A brief review of the anatomy and physiology of the limbic system. Can J Neurological Sci. 2009;36:S84-87.

46. Gramling L, Kapoulea E, Murphy C. Taste perception and caffeine consumption: an fMRI study. Nutrients. 2018;11:34.

47. Svenningsson P. Distribution, biochemistry and function of striatal adenosine A2A receptors. Prog Neurobiol. 1999;59:355-96.

48. Fredholm BB, Arslan G, Halldner L, Kull B, Schulte G, Wasserman $\mathrm{W}$. Structure and function of adenosine receptors and their genes. Naunyn-Schmiedeberg's Arch Pharmacol. 2000;362:364-74.

49. Adenosine FBB. Adenosine receptors and the actions of caffeine. Pharmacol Toxicol. 1995;76:93-101.

50. Mishina M, Ishiwata K. Adenosine receptor PET imaging in human brain. Int Rev Neurobiol. 2014;119:51-69.

51. Kahathuduwa CN, Dhanasekara CS, Chin SH, Davis T, Weerasinghe VS, Dassanayake TL, et al. 1-Theanine and caffeine improve target-specific attention to visual stimuli by decreasing mind wandering: a human functional magnetic resonance imaging study. Nutr Res. 2018;49:67-78.

52. Brunyé TT, Mahoney CR, Lieberman HR, Taylor HA. Caffeine modulates attention network function. Brain Cogn. 2010;72:181-8.

53. Halassa MM, Kastner S. Thalamic functions in distributed cognitive control. Nat Neurosci. 2017;20:1669-79.

54. Baumann O, Borra RJ, Bower JM, Cullen KE, Habas C, Ivry RB, et al. Consensus Paper: the role of the cerebellum in perceptual processes. Cerebellum. 2015;14:197-220.

55. Richards G, Smith A. Caffeine consumption and self-assessed stress, anxiety, and depression in secondary school children. J Psychopharmacol. 2015;29:1236-47.

56. Kolesar TA, Bilevicius E, Wilson AD, Kornelsen J. Systematic review and meta-analyses of neural structural and functional differences in generalized anxiety disorder and healthy controls using magnetic resonance imaging. NeuroImage Clin. 2019;24:102016.

57. Koch SBJ, van Zuiden M, Nawijn L, Frijling JL, Veltman DJ, Olff M. Aberrant resting-state brain activity in posttraumatic stress disorder: a meta-analysis and systematic review: theoretical review: brain activity in PTSD during rest. Depress Anxiety. 2016;33:592-605.

58. Xu J, Van Dam NT, Feng C, Luo Y, Ai H, Gu R, et al. Anxious brain networks: a coordinate-based activation likelihood estimation meta-analysis of resting-state functional connectivity studies in anxiety. Neurosci Biobehav Rev. 2019;96:21-30.

59. Yacoubi ME, Ledent C, Parmentier M, Costentin J, Vaugeois JM. The anxiogenic-like effect of caffeine in two experimental procedures measuring anxiety in the mouse is not shared by selective A2A adenosine receptor antagonists. Psychopharmacology. 2000;148:153-63.

60. Yin Y-Q, Zhang C, Wang J-X, Hou J, Yang X, Qin J. Chronic caffeine treatment enhances the resilience to social defeat stress in mice. Food Funct. 2015;6:479-91. 